little is known about how patients recognise an exacerbation. This study aims to identify the signs and symptoms that prompt patients to seek medical attention and also to identify which signs and symptoms health professionals expect patients to consider.

Methods A Factorial Survey was used to create clinical vignettes describing a variety of signs and symptoms of an exacerbation. A total of 30 patients with Chronic Obstructive Pulmonary Disease (mean \%FEV 138.9 ) and 47 health professionals (14 GPs, 19 Specialist Nurses and 15 Respiratory Consultants/Registrars) completed a unique random set of vignettes. A total of 600 patient and 960 health professional vignettes were analysed using multiple regression analysis.

Results There are substantial variations in the symptoms which patients regard as important prompts for action, compared to health professional expectations. Patients rely mainly on the colour and purulence of sputum $(p<0.001)$ as a cue for all possible actions, whereas health professionals expected patients to consider a variety of symptoms.

Conclusion Many symptoms health professionals regard as important are not used to by patients to guide their decisions. As there is increasing focus on patients self-managing their disease, health professionals should exploit the symptoms patients already rely on rather than trying to change their behaviour.

\section{P211 EXACERBATION FREQUENCY AND MAINTENANCE TREATMENT OF COPD IN UK CLINICAL PRACTICE}

doi:10.1136/thoraxjnl-2011-201054c.211

${ }^{1} \mathrm{M}$ Thomas, ${ }^{2} \mathrm{~A}$ Radwan, ${ }^{3} \mathrm{C}$ Stonham, ${ }^{4} \mathrm{~S}$ Marshall. ${ }^{1}$ University of Aberdeen, Aberdeen, UK; ${ }^{2}$ Novartis Pharmaceuticals, Frimley, UK; ${ }^{3}$ Minchinhampton Surgery, Stroud, UK; ${ }^{4} \mathrm{pH}$ Associates, Marlow, UK

Introduction and Objectives COPD exacerbations are associated with high morbidity, mortality and costs. Prevention of exacerbations is recommended as a key goal of COPD management. Pharmacotherapy shown to reduce exacerbation frequency includes long acting beta agonists (LABA), long acting antimuscarinics (LAMA) and inhaled corticosteroids (ICS). There have been suggestions of a "frequent exacerbator" phenotype across severity.

Methods Retrospective observational study of 511 patients with COPD diagnosed during or before 2007. Three years' data per patient on exacerbation frequency, therapies and resource use were collected by trained researchers from routine medical records in 10 general practices in England. COPD severity was defined as: very severe $\left(\mathrm{FEV}_{1}<30 \%\right.$ predicted, $\left.\mathrm{n}=52\right)$, severe $(30-49 \%, \mathrm{n}=145)$ and moderate $(50-79 \%, n=314)$. Exacerbation frequency was annualised and stratified by: 0/year ("non-exacerbators"), $>0<3$ /year and $=3$ / year ("frequent exacerbators").

Results Mean (SD) annual exacerbation frequency: 1.1 (1.1) in moderate, $1.7(1.6)$ in severe and $2.2(2.0)$ in very severe COPD. 69 (14\%) were frequent exacerbators and 97 (19\%) were non-exacerbators. The proportion of frequent exacerbators increased with severity ( $9 \%$ moderate, $19 \%$ severe, $29 \%$ very severe). $14 \%$ did not receive any $\mathrm{LABA}, 25 \%$ any LAMA and $12 \%$ any ICS medication during the 3 -year study period. "Triple therapy" with all three classes was received by only $67 \%$ of the frequent exacerbators. The percentage of patients with zero exacerbations/year fell as severity increased (25\% moderate, $11 \%$ severe, $8 \%$ very severe). $61 \%$ received a LABA and/or LAMA with ICS (56\% moderate, $75 \%$ severe and $100 \%$ very severe). $32 \%$ did not receive ICS during the study period (35\% moderate, $25 \%$ severe and $0 \%$ very severe). The median number of primary care visits was 1.33 for patients with $0,2.67$ for $>0<3$ and 6.67 for $=3$ exacerbations/year.

Conclusions The "frequent exacerbator" phenotype occurs in patients of all levels of severity in community practice in the UK. Health resource use is high in frequent exacerbators. These patients frequently do not receive optimum therapy. The exacerbation history can potentially guide maintenance treatment of patients at all levels of COPD severity.

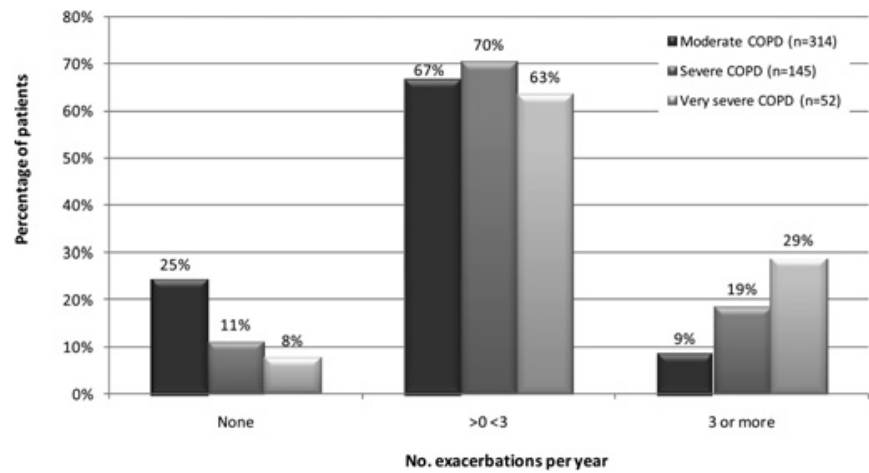

Abstract P211 Figure 1 Distribution of number of COPD exacerbations per year by severity of disease.

\section{P212 THE DECAF SCORE: PREDICTING IN-HOSPITAL MORTALITY IN ACUTE EXACERBATIONS OF COPD}

doi:10.1136/thoraxjnl-2011-201054c.212

${ }^{1} \mathrm{~J}$ Steer, ${ }^{2} \mathrm{G}$ J Gibson, ${ }^{1} \mathrm{~S}$ C Bourke. ${ }^{1}$ Department of Respiratory Medicine, North Tyneside General Hospital, Northumbria Healthcare NHS Foundation Trust, North Shields, UK; ${ }^{2}$ Newcastle University, Newcastle-upon-Tyne, UK

Background Despite the often poor outcome of patients hospitalised with acute exacerbations of COPD (AECOPD), it is difficult accurately to identify those at high risk of mortality. To aid prognostication in AECOPD, we have developed a simple, easily memorable and effective tool, based on clinical data available shortly after admission.

Methods Consecutive patients hospitalised with AECOPD were recruited, with clinical and demographic data collected at admission. In-hospital mortality data were collected from hospital records. Variables were dichotomised and the strongest independent predictors of mortality were identified by logistic regression analysis. Tool performance was assessed using ROC curve analysis.

Results 920 patients were recruited: mean (SD) age was 73.1 (10.0) years, with $53.9 \%$ female; most had severe airflow obstruction ( $\mathrm{FEV}_{1} 43.6$ (17.2) \% predicted) and were of normal weight (BMI 24.6 $\left.(6.3) \mathrm{kg} / \mathrm{m}^{2}\right) .32 .5 \%$ of patients had coexistent consolidation and 199 $(21.6 \%)$ received assisted ventilation during their hospital stay. 96 $(10.4 \%)$ patients died in-hospital. In descending order of strength, the factors independently predicting mortality were: the extended MRC Dyspnoea Scale (eMRCD) ${ }^{1}$; coexistent radiographic consolidation; eosinopenia $\left(<0.05 \times 10^{9} / 1\right) ; \mathrm{pH}<7.3$; atrial fibrillation; cough effectiveness; albumin $<36 \mathrm{~g} / \mathrm{dl}$; age $=80$ years; cerebrovascular disease; and $\mathrm{BMI}<18.5 \mathrm{~kg} / \mathrm{m}^{2}$. The strongest five variables were selected to form the DECAF (Dyspnoea, Eosinopenia, Consolidation, Acidaemia, atrial Fibrillation) score (Abstract P212

Abstract P212 Table 1 DECAF score and in-hospital mortality

\begin{tabular}{lllll}
\hline DECAF score & $\mathbf{n}$ & $\begin{array}{l}\text { In-hospital } \\
\text { mortality, } \%\end{array}$ & Sensitivity* & Specificity* \\
\hline 0 & 201 & 0.5 & 1 & 0 \\
1 & 291 & 2.1 & 0.99 & 0.24 \\
2 & 226 & 8.4 & 0.93 & 0.59 \\
3 & 125 & 24 & 0.73 & 0.84 \\
4 & 57 & 45.6 & 0.42 & 0.96 \\
5 & 20 & 70 & 0.15 & 0.99 \\
6 & 0 & NA & NA & NA \\
\hline
\end{tabular}

*For DECAF score $\geq$ that stated. 
table 1). Each predictor was assigned a score of 1 (present) or 0 (absent), except for eMRCD score which could be 0,1 or 2 , giving a maximum DECAF score of 6. The DECAF score showed good performance for the prediction of in-hospital mortality (area under ROC curve $=0.858$, 95\% CI 0.82 to 0.89 ), and was a stronger predictor $(p<0.0001)$ than either the APACHE (AUROC $=0.727)$ or CAPS (AUROC $=0.710$ ) prognostic scores. In patients with coexistent consolidation $(n=299)$, DECAF was a stronger predictor of mortality than CURB-65 (AUROC $=0.77$ vs $0.66, p=0.0064$ ).

Conclusion The DECAF score is a strong predictor of in-hospital mortality and may improve the prognostication of patients hospitalised with AECOPD. External validation is required before recommending widespread application.

\section{REFERENCE}

1. Steer J. BTS Winter Meeting. London, 2010.

\section{P213 LONGITUDINAL CHANGES IN THE RATE AND MEAN AGE OF INCIDENCE AND PREVALENCE OF COPD IN THE UK, 2000-2009}

doi:10.1136/thoraxjnl-2011-201054c.213

G James, I Petersen, G Donaldson, W Wedzicha. University College London, London, $U K$

Introduction Since the NICE guidelines in 2004 and introduction of the Quality and Outcomes Framework (QOF) in 2004, the prevalence of COPD seen in general practice has increased (Smith 2008). ${ }^{1}$ We have investigated whether this trend has continued until 2009, and explored whether this can be explained by change in mean age at diagnosis and death.

Methods We identified all patients aged between 35 and 89 years $(\mathrm{n}=2173494$, mean age $=56,49 \%$ male) in The Health Improvement Network (THIN) primary care database. From this cohort of
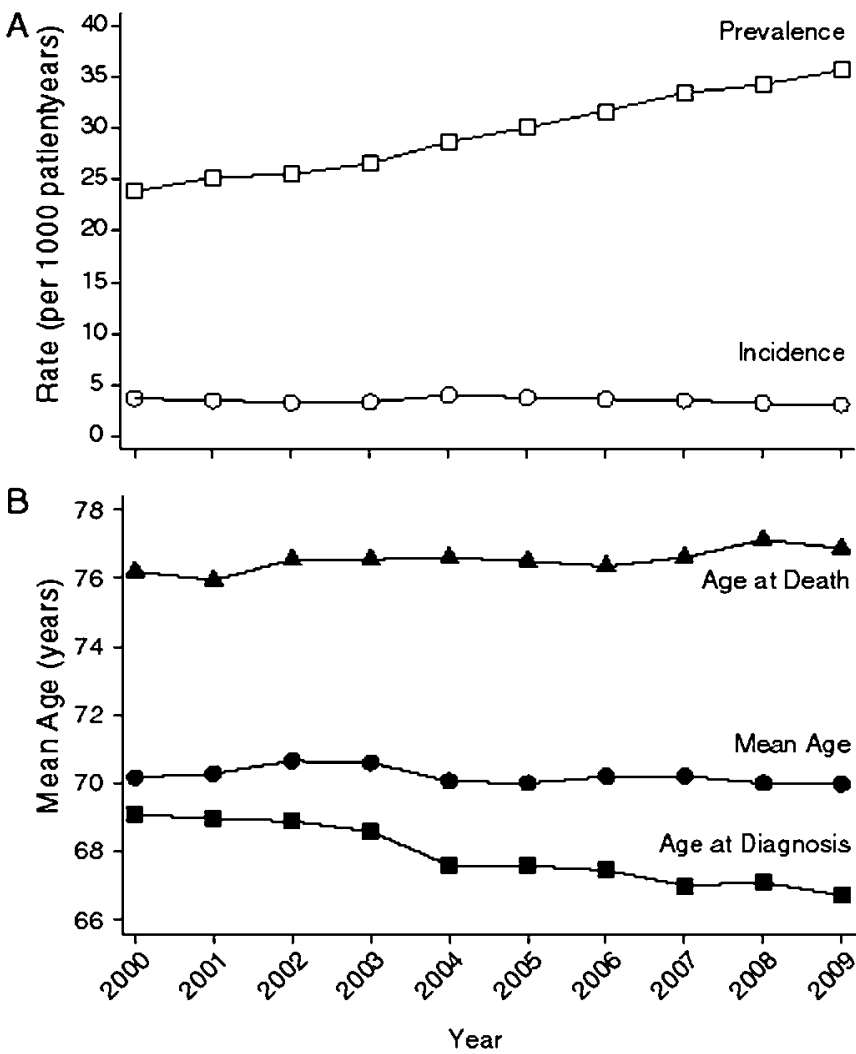

Abstract P213 Figure 1 patients we identified patients with a diagnosis of COPD using the criteria for $\mathrm{QOF}$. We calculated annual incidence and prevalence rates, and mean age of patients with COPD, first COPD diagnosis and death between 2000 and 2009 .

Results In total, 53379 (2.5\%) of the patients were diagnosed with COPD. The prevalence of COPD increased by $50 \%$ over the 10 -year period, from 24 cases per 1000 patient years in 2000 to 36 in 2009 (Abstract P213 figure 1A). However, the diagnosis of new COPD cases remained fairly constant $(p=0.295)$, at 3.5 cases per 1000 patient years (Abstract P213 figure 1A). The mean age at first COPD diagnosis (incidence) decreased significantly $(p<0.001)$ by 2 years and 5 months from 69 years and 1 months in 2000 to 66 years and 8 months in 2009 (Abstract P213 figure 1B). The mean age at death of COPD patients increased significantly $(\mathrm{p}=0.008)$ by 9 months from 76 years and 2 months in 2000 to 76 years and 11 months in 2009 (Abstract P213 figure 1B). Whereas the mean age of prevalent patients remained fairly constant $(\mathrm{p}=0.098)$ over the period, varying between 70 years and 70 years and 8 months (Abstract P213 figure 1B).

Conclusion We found that over the last decade, the average age of patients with COPD has remained relatively constant at around 70 years. COPD is increasingly being diagnosed at a younger age and patients are living longer, which may in part explain the $50 \%$ rise in COPD prevalence.

\section{REFERENCE}

1. Smith CJ, Gribbin J, Challen KB, et al. The impact of the 2004 NICE guideline and 2003 General Medical Services contract on COPD in primary care in the UK. OJM 2008; 101:145-3

\section{P214 ACUTE EXACERBATIONS OF COPD: A REVIEW OF RESUSCITATION STATUS AND ASSOCIATIONS WITH PROGNOSTIC FACTORS IN HOSPITAL ADMISSIONS}

doi:10.1136/thoraxjnl-2011-201054c.214

B Rudran, L Idris, C Childs, F Riccio, S Loganathan, T J Shaw. Royal Bournemouth Hospital, Bournemouth, UK

Introduction Acute exacerbations of COPD are among the most common reasons for hospital admission in the UK. Exacerbations can lead to respiratory failure requiring ventilatory support, and so decisions regarding "escalation" or "ceilings" of treatment are often made early in admission. Such decisions on intubation are inevitably linked to decisions regarding resuscitation status. Prognostic factors should be used when making these decisions and $\mathrm{FEV}_{1}$ should not be used exclusively. We reviewed admissions with exacerbations of COPD, categorised by resuscitation status, to see if there were differences in prognostic features between groups.

Methods 53 acute admissions with exacerbations of COPD were reviewed between 1 December 2010 and 31 January. Groups were divided by resuscitation status: documented decision not to attempt resuscitation (DNR), no documented decision ( $\mathrm{NoD}$ ) and documented decision for resuscitation (ForR). Data were collected on individual prognostic factors; we then calculated prognostic indices against known criteria. $P$ values were calculated using Mann-Whitney U test.

Results The significant findings were that patients in the DNR group had lower $\mathrm{FEV}_{1}$, more likely to have home oxygen and had a poorer functional status. Age, comorbidity, BMI and previous ITU admission were not found to be significantly different between the groups. When the prognostic indices were calculated the patients in the DNR group were found to have higher scores, correlating with poorer prognosis.

Conclusions From the results we can infer which factors are being used for resuscitation decisions in patients with COPD. Functional status and home oxygen are most relied upon with $\mathrm{FEV}_{1}$ somewhat less so. Age, comorbidity and BMI are not being taken into account, 